\title{
Diagnostic Agreement between Prehospital Emergency and In-Hospital Physicians
}

\author{
Nikolai Ramadanov $\mathbb{D}^{\mathrm{D}},{ }^{1,2}$ Roman Klein, ${ }^{3}$ Fabian Laue, ${ }^{2}$ and Wilhelm Behringer ${ }^{1}$ \\ ${ }^{1}$ Center for Emergency Medicine, University Hospital Jena, Friedrich Schiller University Am Klinikum 1, 07747 Jena, Germany \\ ${ }^{2}$ Clinic for Reconstruction and Trauma Surgery, Ernst von Bergmann Hospital Charlottenstr. 72, 14467 Potsdam, Germany \\ ${ }^{3}$ Orthopaedics, Trauma Surgery and Sports Traumatology, Marienhausklinikum Hetzelstift, Stiftstr. 10, 67434 Neustadt, Germany
}

Correspondence should be addressed to Nikolai Ramadanov; nikolai.ramadanov@gmail.com

Received 10 January 2019; Revised 20 February 2019; Accepted 26 March 2019; Published 24 April 2019

Academic Editor: Chak W. Kam

Copyright (C) 2019 Nikolai Ramadanov et al. This is an open access article distributed under the Creative Commons Attribution License, which permits unrestricted use, distribution, and reproduction in any medium, provided the original work is properly cited.

\begin{abstract}
Background. The aim of the study was to determine the diagnostic agreement between the discharge diagnosis and the suspected diagnosis by the prehospital emergency physician and to run a sensitivity analysis of the most common diagnoses by the prehospital emergency physician. Methods. The diagnostic agreement was determined by a systematic comparison of the discharge diagnosis with suspected diagnosis by the prehospital emergency physician in a period of 24 months at the emergency medical services in Bad Belzig. The diagnostic agreement of the 13 most common discharge diagnoses was compared to the remaining diagnostic agreement. The results were tested for statistical significance using the chi-squared test. Results. In $64.1 \%$ of cases included, a diagnostic agreement occurred. There was a high proportion of diagnostic agreement for hypoglycemia (97\%), atrial fibrillation (87\%), cramping seizure (86\%), hypertensive crisis (85.5\%), and syncope (81\%). There was a low proportion of diagnostic agreement for chest wall pain (27\%), pneumonia (32\%), and cardiac decompensation (53\%). Conclusions. Our attention in practice and emergency medical courses should be directed to chest pain patients and the main symptom of dyspnea, because of the high proportion of incorrect diagnoses by the prehospital emergency physician. It should be noted that $92 \%$ of incorrectly diagnosed chest wall pain cases were overestimated with an acute coronary syndrome.
\end{abstract}

\section{Introduction}

Due to the lack of laboratory-chemical examinations and imaging diagnostics in the emergency medical services (EMS), the correct prehospital diagnosis is a challenge for the prehospital emergency physician. Nevertheless, due to the often life-saving nature of prehospital missions, the correct diagnosis is important to the patient outcome. There are several studies done about the diagnostic agreement (dA) in the EMS and in the emergency department (ED) [1-4]. The results for dA showed a very broad spectrum (46.5\%$90 \%)$. It should be noted that in these studies the methods for determining $\mathrm{dA}$ were different. A breakdown and sensitivity analysis of the most common diagnoses by the prehospital emergency physician has not yet been performed. It is of utmost importance for emergency medical science to know which clinical pictures have a low diagnostic agreement in prehospital missions.

The aim of our study was to determine the dA between emergency mission-related discharge diagnoses and suspected diagnoses by the prehospital emergency physician and to perform a sensitivity analysis of the most common emergency mission-related discharge diagnoses.

\section{Study Design and Examination Methods}

2.1. Data Collection. All prehospital emergency physician's patient care reports (DIVI protocol 4.2) of the EMS in Bad Belzig and the corresponding discharge summaries from the hospital information system in Bad Belzig (SAP Version 7400.1.0.1093 and Cerner Soarian Clinicals Version 4.1) in the 
period from July 1, 2013, to June 30, 2014, and from January 1,2015 , to December 31, 2015, were examined. Further discharge summaries were included from neighboring hospitals (Klinikum Ernst von Bergmann Potsdam, Asklepios Fachklinik Brandenburg, Städtisches Krankenhaus Brandenburg, Johanniter Krankenhaus in Fläming Treuenbrietzen). The study was approved by the ethical committee of the University of Jena (No. 4522-08/15/15).

2.2. Diagnostic Agreement. First, all prehospital missionrelated discharge diagnoses from the 1055 included cases were determined. In case that the discharge diagnosis from the discharge summaries for the corresponding mission could be confirmed in the suspected diagnoses from the patient care reports by the prehospital emergency physician, a dA occurred. In case that the discharge diagnosis from the discharge summaries for the corresponding mission could not be confirmed in the suspected diagnosis from the patient care reports by the prehospital emergency physician, a dA did not occur. This systematic comparison established the dA for all 1378 prehospital mission-related discharge diagnoses. It was done by two experienced emergency physicians (N. Ramadanov and F. Laue) independently from each other using the ICD 10 coding $(\kappa=0,95)$. In divergent cases a third emergency physician (W. Behringer) helped find the correct adjudication.

2.3. Statistics. As part of a sensitivity analysis, the dA of the 13 most common prehospital mission-related hospital discharge diagnoses was tested for statistically significant differences compared to the remaining $\mathrm{dA}$. The chi-square test was used with a significance level of $p=0.05$. Statistical calculations were performed using IBM SPSS Statistics 19 for Windows.

\section{Results}

3.1. Exclusion of Cases. 705 patient care reports were excluded from the study for the following reasons: ambulant treatment in the emergency department; prehospital treatment, lack of admission to the ED; lack of recorded emergency diagnosis; death of the patient during the mission or incorrect/unreadable patient data (see Figure 1).

3.2. Patients and Prehospital Emergency Physicians. The average age of the 1055 patients was 70 years (min. 1, max 100 years). 493 of them were male and 562 were female. $64.5 \%$ of the prehospital missions were carried out by internist prehospital emergency physicians, $27 \%$ by surgical, $7 \%$ by general practitioners, and $1.5 \%$ by anesthetic emergency physicians. $70 \%$ of the prehospital missions were carried out by resident physicians and $30 \%$ by specialist physicians. $33 \%$ of the prehospital missions were carried out by prehospital emergency physicians with a higher approval for emergency medicine ("Zusatzbezeichnung Notfallmedizin") and $67 \%$ of the prehospital missions by prehospital emergency physicians with a lower approval for emergency medicine ("Fachkunde Rettungsdienst").

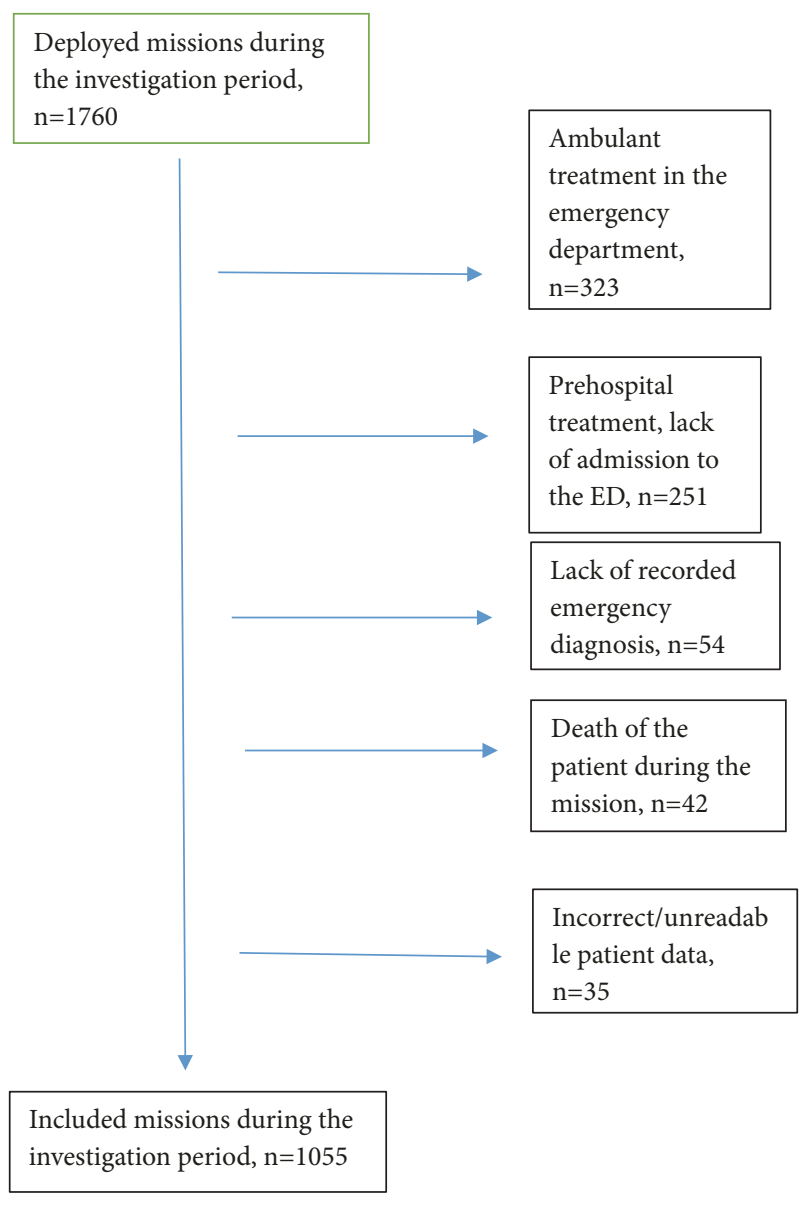

FIGURE 1: Inclusion chart.

3.3. Spectrum of Discharge Diagnoses. At 1055 included cases, a total of 1378 prehospital mission-related hospital discharge diagnoses were formulated after emergency medical admission. In 238 cases there were made 2 discharge diagnoses for one patient, in 38 cases 3 discharge diagnoses and in 3 cases 4 discharge diagnoses for one patient. Table 1 shows the 13 most common prehospital mission-related discharge diagnoses after emergency medical admission and Figure 2 the distribution of discharge diagnoses by specialty.

3.4. Determined Diagnostic Agreement and Sensitivity Anal$y$ sis. A dA occurred in $64.1 \%$ and did not occur in $35.9 \%$ of cases. The results for the sensitivity analysis of the 13 most common prehospital mission-related hospital discharge diagnoses are listed in Table 2.

3.5. Accumulation of $d A$. An accumulation of $\mathrm{dA}$ among the 13 most common prehospital mission-related hospital discharge diagnoses was determined for hypoglycemia with 97\% (Chi-square $=16.69 ; \mathrm{DF}=2 ; \mathrm{p}=0,01$ ), arrhythmia absoluta with $87 \%$ (Chi-square $=9.36 ; \mathrm{DF}=1 ; \mathrm{p}=0,01$ ), seizure with $86 \%$ (Chi-square $=7.84 ; \mathrm{DF}=1 ; \mathrm{p}=0,01$ ), hypertensive crisis with $85,5 \%$ (Chi-square $=25,57$; $\mathrm{DF}=1$; 
TABle 1: The 13 most common prehospital mission-related hospital discharge diagnoses by the prehospital emergency physician $(\mathrm{n}=1378)$.

\begin{tabular}{|c|c|c|c|}
\hline ICD & Designation & $\%$ & Number \\
\hline I10.91 & Hypertensive crisis & 8.5 & 117 \\
\hline I50.9 & Cardiac decompensation & 6.8 & 94 \\
\hline R55, I95.9 & Syncope, hypotonic dysregulation & 6.1 & 84 \\
\hline E86 & Dehydration & 5.2 & 71 \\
\hline I21.9 & Acute coronary syndrome & 4.1 & 57 \\
\hline J15.9 & Pneumonia & 3.8 & 53 \\
\hline J44.09 & Exacerbated COPD & 3.4 & 47 \\
\hline I48.9 & Arrhythmia absoluta & 2.8 & 39 \\
\hline $\mathrm{I} 20.0$ & Stable angina pectoris & 2.6 & 36 \\
\hline G40.9, R56.8, R56.0 & Seizure & 2.6 & 36 \\
\hline R07.4, G58.8 & Chest wall pain & 2.4 & 33 \\
\hline E16.2/E15 & Hypoglycemia/hypoglycemic coma & 2.2 & 31 \\
\hline I63.9 & Stroke & 1.9 & 26 \\
\hline
\end{tabular}

TABLE 2: Sensitivity analysis of the most common prehospital mission-related hospital discharge diagnoses by the prehospital emergency physician.

\begin{tabular}{lccc}
\hline Designation & Yes & Number of dA (n) & No \\
\hline Diagnoses total & $64,1 \%(883)$ & $35,9 \%(495)$ & p \\
Hypertensive crisis & $85,5 \%(100)$ & $14,5 \%(17)$ & 0,01 \\
Cardiac decompensation & $53 \%(50)$ & $47 \%(44)$ & 0,02 \\
Syncope, hypotonic dysregulation & $81 \%(68)$ & $19 \%(16)$ & 0,01 \\
Dehydration & - & - & 0,89 \\
Acute coronary syndrome & - & - & 0,88 \\
Pneumonia & $32 \%(17)$ & $68 \%(36)$ & 0,01 \\
Exacerbated COPD & - & - & 0,12 \\
Arrhythmia absoluta & $87 \%(34)$ & $13 \%(5)$ & 0,01 \\
Stable angina pectoris & $81 \%(29)$ & $19 \%(7)$ & 0,04 \\
Seizure & $86 \%(31)$ & $14 \%(5)$ & 0,01 \\
Chest wall pain & $27 \%(9)$ & $73 \%(24)$ & 0,01 \\
Hypoglycemia/hypoglycemic Coma & $3 \%(1)$ & 0,01 \\
Stroke & $97 \%(30)$ & - & 0,57 \\
\hline
\end{tabular}

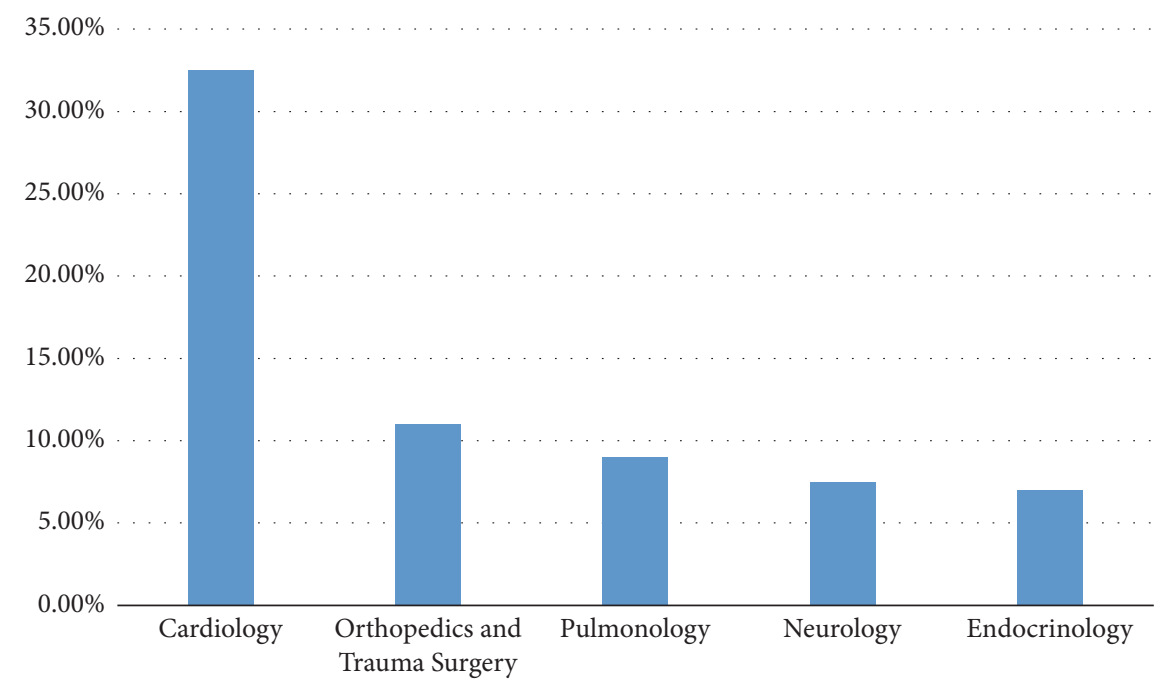

FIGURE 2: Distribution of the prehospital mission-related hospital discharge diagnoses by the prehospital emergency physician, summarized by specialty. 
$\mathrm{p}=0,01)$, and syncope with $81 \%$ (Chi-square $=11,15 ; \mathrm{DF}=1$; $\mathrm{p}=0,01)$.

3.6. Lack of $d A$. Table 4 showed a low proportion of $\mathrm{dA}$ for chest wall pain with $27 \%$ compared to the dA of the rest of diagnoses (chi-square $=19.8, \mathrm{DF}=1, \mathrm{p}=0.01$ ). In $92 \%$ of cases of missing $\mathrm{dA}$ chest wall pain was considered incorrectly as acute coronary syndrome. Table 5 showed a low proportion of $\mathrm{dA}$ for pneumonia with $68 \%$ compared to the $\mathrm{dA}$ of the rest of diagnoses (chi-square $=24.34, \mathrm{DF}=1, \mathrm{p}=0.01$ ). In $30 \%$ of cases of missing $\mathrm{dA}$, pneumonia was considered incorrectly as pulmonary edema and cardiac decompensation. Table 6 showed a low proportion of $\mathrm{dA}$ for cardiac decompensation with $47 \%$ compared to the $\mathrm{dA}$ of the rest of diagnoses (chisquare $=5.12, \mathrm{DF}=1, \mathrm{p}=0.02$ ). In $22 \%$ of cases of missing $\mathrm{dA}$, cardiac decompensation was considered incorrectly as exacerbated COPD.

\section{Discussion}

The most common prehospital mission-related hospital discharge diagnoses were made in cardiopulmonary diseases (42\%), followed by the specialties orthopedics and trauma surgery (11\%), neurology (8\%), and endocrinology (7.5\%). The most common prehospital mission-related hospital discharge diagnoses were hypertensive crisis $(8.5 \%)$, cardiac decompensation $(6.8 \%)$, syncope $(6.1 \%)$, dehydration $(5.2 \%)$, and acute coronary syndrome $(4.1 \%)$.

Calculating the $\mathrm{dA}$, it was possible to estimate retrospectively the correctness of the suspected diagnosis by the prehospital emergency physician. Based on 1055 included missions, our findings showed that the suspected diagnosis was correct in $64.1 \%$ of the cases. The sensitivity analysis of the 13 most common prehospital mission-related hospital discharge diagnoses showed a significantly high proportion of correct diagnoses for hypoglycemia (97\%), arrhythmia absoluta $(87 \%)$, seizure $(86 \%)$, hypertensive crisis $(85.5 \%)$, and syncope $(81 \%)$ and a significantly high proportion of incorrect diagnoses for chest wall pain (73\%), pneumonia (68\%), and cardiac decompensation (47\%).

4.1. Accumulation of Correct Suspected Diagnoses by the Prehospital Emergency Physician. For diseases with a very remarkable clinical picture such as seizure and syncope, the accumulation of correct suspected diagnosis is understandable. The accumulation of correct suspected diagnoses by the prehospital emergency physician for hypoglycemia, hypertensive crisis, and arrhythmia absoluta can be explained by the fact that there is preclinical possibility for precise diagnostics (blood glucose measurement, blood pressure measurement, 12-channel ECG) in these diseases. The impact of the lack of laboratory and imaging diagnostics in prehospital medicine can be seen in the comparison of our present results with the study by Dormann with similar methods for the determination of the dA. Among other things, the admission diagnosis from the emergency department was compared to the hospital discharge diagnosis in his study [3]. In 4321 hospitalized patients, the $\mathrm{dA}$ was $71 \%$, well above the dA of the present study (64.1\%), which can be explained simply by the lack of opportunities for laboratory-chemical examinations and imaging diagnostics.

4.2. Lack of Correct Suspected Diagnoses by the Prehospital Emergency Physician for Chest Wall Pain. The chest wall pain represents a prehospital diagnostic challenge with a low proportion of $27 \%$ of correct suspected diagnoses by the prehospital emergency physician. This was already discovered in numerous other studies [7, 8]. Our findings showed that in $92 \%$ of the cases of chest wall pain the prehospital suspected diagnoses by the prehospital emergency physician were considered as an acute coronary syndrome and were accordingly mistreated with drugs (Heparin, acetylsalicylic acid). "Most patients with "pectanginal" symptoms do not suffer from a life-threatening heart disease" [9]. In a study with 1212 "chest pain patients", who visited a GP practice, only $3.6 \%$ had an acute coronary syndrome [10]. Obviously the prehospital chest pain needs more attention being a prehospital differential diagnostic challenge. A more accurate medical history and physical examination should improve the correctness of the suspected diagnosis. Table 3 is reproduced from McConaghy et al. (2013) [under the Creative Commons Attribution License/https://www.aafp.org/ afp/2013/0201/p177.html]. It shows a possible differential diagnostic procedure for chest pain patients that was created after evaluation of 13 other studies [5, 6, 8, 11-14].

4.3. Lack of Correct Diagnoses for Main Symptom "Dyspnea". Pneumonia with 32\% and cardiac decompensation with 53\% showed a low proportion of correct suspected diagnoses by the prehospital emergency physician. Furthermore a high proportion of diseases from the cardiopulmonary area with common main symptom "dyspnea" was noticed during the evaluation of the prehospital mission-related hospital discharge diagnoses. So the main symptom "dyspnea" presents a prehospital diagnostic challenge because of its diverse causes. This was already determined in other studies [10, 15].

4.4. Limitations. There is a certain degree of subjectivity in the determination of $\mathrm{dA}$ in this study as well as in other studies, since the choice of different methods may lead to deviations in the results. Since there are differences in the EMS system over the whole country, the described EMS location is not sufficiently representative. A geographically varying distribution of the diagnoses is conceivable. Transferring the results to other EMS locations and regions is therefore difficult.

\section{Conclusion}

(i) Special attention in the prehospital mission and during the education of prehospital emergency physicians should be paid to chest pain patients due to the accumulation of misdiagnosis, as $92 \%$ of incorrectly diagnosed patients with chest wall pain were overestimated and mistreated as cases with acute coronary syndrome. 
TABle 3: Differential diagnosis of chest pain, adapted from Conaghy et al.

\begin{tabular}{|c|c|c|c|}
\hline Acute myocardial infarction & Chest pain radiates to both arms & 7.1 & 0.67 \\
\hline & Third heart sound on auscultation & 3.2 & 0.88 \\
\hline & Hypotension & 3.1 & 0.96 \\
\hline Chest wall pain & $\begin{array}{l}\text { At least two of the following findings: localized muscle } \\
\text { tension; stinging pain; pain reproducible by palpation; } \\
\text { absence of cough }\end{array}$ & 3.0 & 0.47 \\
\hline Gastroesophageal reflux disease & $\begin{array}{l}\text { Burning retrosternal pain, acid regurgitation, sour or } \\
\text { bitter taste in the mouth; one-week trial of high-dose } \\
\text { proton pump inhibitors relieves symptoms }\end{array}$ & 3.1 & 0.30 \\
\hline Panic disorder/anxiety state & $\begin{array}{l}\text { Single question: in the past four weeks, have you had an } \\
\text { anxiety attack (suddenly feeling fear or panic)? }\end{array}$ & 4.2 & 0.09 \\
\hline Acute thoracic aortic dissection & $\begin{array}{l}\text { Acute chest or back pain and a pulse differential in the } \\
\text { upper extremities }\end{array}$ & 5.3 & NA \\
\hline
\end{tabular}

Note: The higher the LR is above 1, the better it rules in disease (greater than 10 is considered good). Conversely, the lower the LR is below 1 , the better it rules out disease (less than 0.1 is considered good). $\mathrm{LR}+=$ positive likelihood ration; LR- = negative likelihood ratio; NA $=$ not available. Information is from references [5] through [6].

TABLE 4: dA for "chest wall pain" compared to the remaining diagnoses.

\begin{tabular}{lccc}
\hline & \multicolumn{2}{c}{ Number of dA (n) } & p \\
& Yes & No & \\
\hline Chest wall pain & $27 \%(9)$ & $73 \%(24)$ & \multirow{2}{*}{0,01} \\
Rest & $65 \%(874)$ & $35 \%(468)$ & \\
\hline
\end{tabular}

TABLE 5: dA for "pneumonia" compared to the remaining diagnoses.

\begin{tabular}{lccc}
\hline & \multicolumn{2}{c}{ Number of dA (n) } & p \\
& Yes & No & \\
\hline Pneumonia & $32 \%(17)$ & $68 \%(36)$ & \multirow{2}{*}{0,01} \\
Rest & $65 \%(866)$ & $35 \%(459)$ & \\
\hline
\end{tabular}

TABLE 6: dA for "cardiac decompensation" compared to the remaining diagnoses

\begin{tabular}{lccc}
\hline & \multicolumn{2}{c}{ Number of dA (n) } & p \\
& Yes & No & \\
\hline $\begin{array}{l}\text { Cardiac } \\
\text { decompensation }\end{array}$ & $53 \%(50)$ & $47 \%(44)$ & 0,02 \\
Rest & $65 \%(833)$ & $35 \%(451)$ & \\
\hline
\end{tabular}

(ii) Special attention in the prehospital mission and during the education of prehospital emergency physicians should be paid as well on cardiopulmonary diseases with the common main symptom "dyspnea" as a diagnostic challenge.

\section{Abbreviations}

dA: Diagnostic agreement

COPD: Chronic obstructive pulmonary disease

DIVI: Deutsche interdisziplinäre Vereinigung

für Intensiv- und Notfallmedizin

EMS: Emergency medical services

ED: Emergency department
GP: $\quad$ General practitioner

IBM SPSS: International Business Machines Corporation Superior Performing Software System

SAP: $\quad$ Systeme, Anwendungen, Produkte.

\section{Data Availability}

The datasets used and/or analysed during the current study are available from the corresponding author upon reasonable request.

\section{Conflicts of Interest}

The authors declare that they have no conflicts of interest.

\section{References}

[1] J. Peter, "Qualität notärztlicher Diagnosen: Ein Vergleich von Fachärzten und Weiterbildungsassistenten der Anästhesie Bd. 4, Medizinische Fakultät der Friedrich-Alexander-Universität, Erlangen-Nürnberg," 2010, http://www.dnb.de.

[2] H. Arntz, S. Klatt, R. Stern, S. Willich, and J. Benecker, "Sind Notarztdiagnosen zuverlässig?" Notfall and Rettungsmed, pp. 12-19, 1997.

[3] H. Dormann, K. Diesch, T. Ganslandt, and E. G. Hahn, "Kennzahlen und Qualitätsindikatoren einer medizinischen Notaufnahme," Deutsches Ärzteblatt International, vol. 107, no. 15, pp. 261-267, 2010.

[4] N. Ramadanov, P. Schlattmann, and W. Behringer, "Übereinstimmung zwischen notärztlicher Verdachtsdiagnose und Entlassungsdiagnose," Notfall and Rettungsmedizin, vol. 21, no. 7, 2018.

[5] A. A. Panju, B. R. Hemmelgarn, G. H. Guyatt, and D. L. Simel, "Is this patient having a myocardial infarction?" Journal of the American Medical Association, vol. 280, no. 14, pp. 1256-1263, 1998.

[6] C. S. Wang, J. M. FitzGerald, M. Schulzer, E. Mak, and N. T. Ayas, "Does this dyspneic patient in the emergency department 
have congestive heart failure?" Journal of the American Medical Association, vol. 294, no. 15, pp. 1944-1956, 2005.

[7] R. R. Bruno, N. Donner-Banzhoff, W. Söllner et al., “The interdisciplinary management of acute chest pain," Deutsches Ärzteblatt International, vol. 112, no. 45, pp. 768-780, 2015.

[8] J. R. McConaghy and R. S. Oza, "Outpatient diagnosis of acute chest pain in adults," American Family Physician, vol. 87, no. 3, pp. 177-182, 2013.

[9] N. Von Lutterotti, "Chest pain units: wo brustschmerzen gezielt 'gefiltert' werden," Deutsches Ärzteblatt, vol. 98, no. 16, pp. 335336, 2001, A-1038/B-878/C-826.

[10] D. Berliner, N. Schneider, T. Welte et al., "The differential diagnosis of dyspnea," Deutsches Ärzteblatt International, vol. 113, no. 49, pp. 834-845, 2016.

[11] S. Bösner, A. Becker, M. A. Hani et al., "Chest wall syndrome in primary care patients with chest pain: presentation, associated features and diagnosis," Journal of Family Practice, vol. 27, no. 4, pp. 363-369, 2010.

[12] J. Zimmerman, "Validation of a brief inventory for diagnosis and monitoring of symptomatic gastrooesophageal reflux," Scandinavian Journal of Gastroenterology, vol. 39, no. 3, pp. 212216, 2004.

[13] W. H. Wang, J. Q. Huang, G. F. Zheng et al., "Is proton pump inhibitor testing an effective approach to diagnose gastroesophageal reflux disease in patients with noncardiac chest pain? a meta-analysis," JAMA Internal Medicine, vol. 165, no. 11, pp. 1222-1228, 2005.

[14] B. Löwe, K. Gräfe, S. Zipfel et al., "Detecting panic disorder in medical and psychosomatic outpatients: Comparative validation of the Hospital Anxiety and Depression Scale, the Patient Health Questionnaire, a screening question, and physicians' diagnosis," Journal of Psychosomatic Research, vol. 55, no. 6, pp. 515-519, 2003.

[15] A. M. Kelly, G. Keijzers, S. Klim et al., "An observational study of dyspnea in emergency departments: the Asia, Australia, and New Zealand Dyspnea in Emergency Departments Study (AANZDEM)," Academic Emergency Medicine, vol. 24, no. 3, pp. 328-336, 2017. 


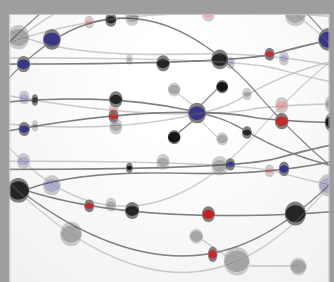

The Scientific World Journal
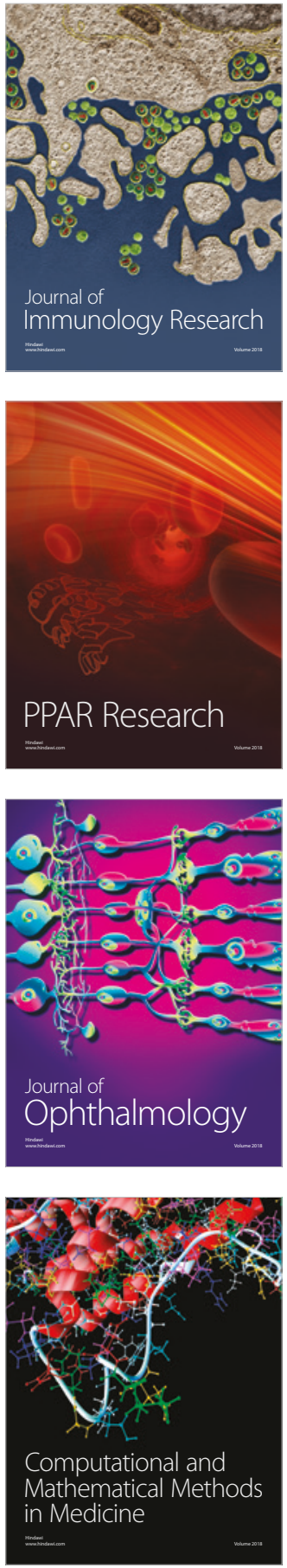

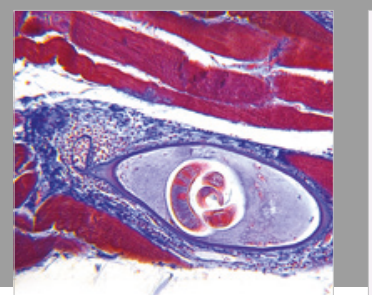

Gastroenterology Research and Practice

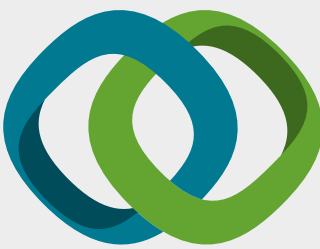

\section{Hindawi}

Submit your manuscripts at

www.hindawi.com
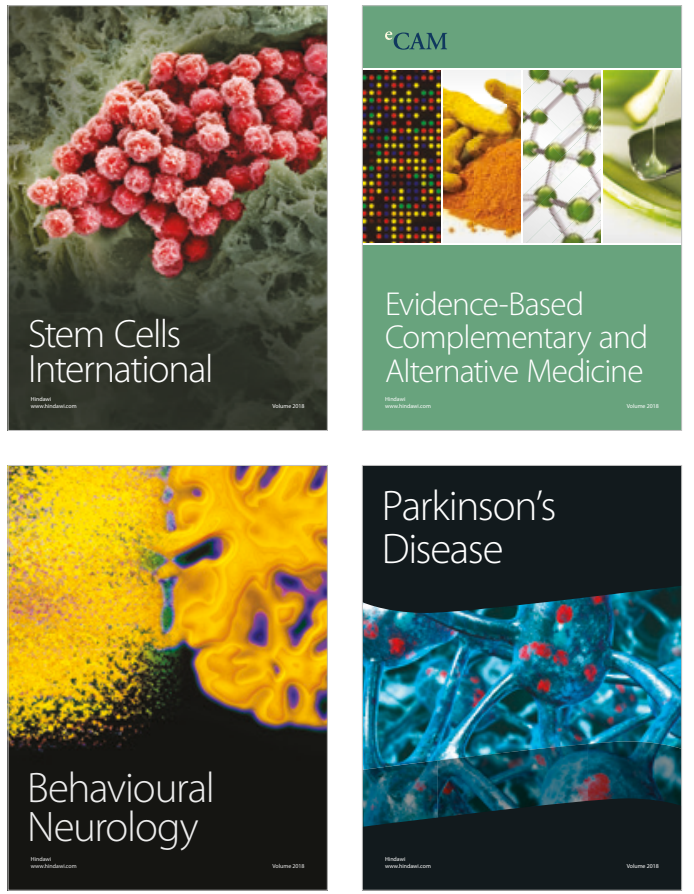

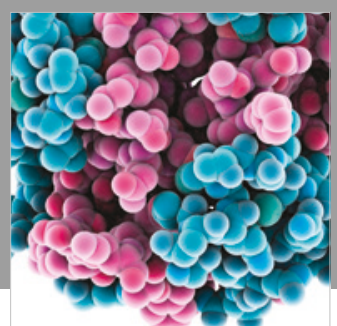

ournal of

Diabetes Research

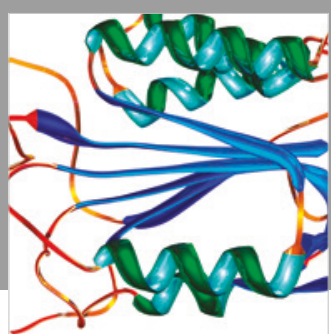

Disease Markers
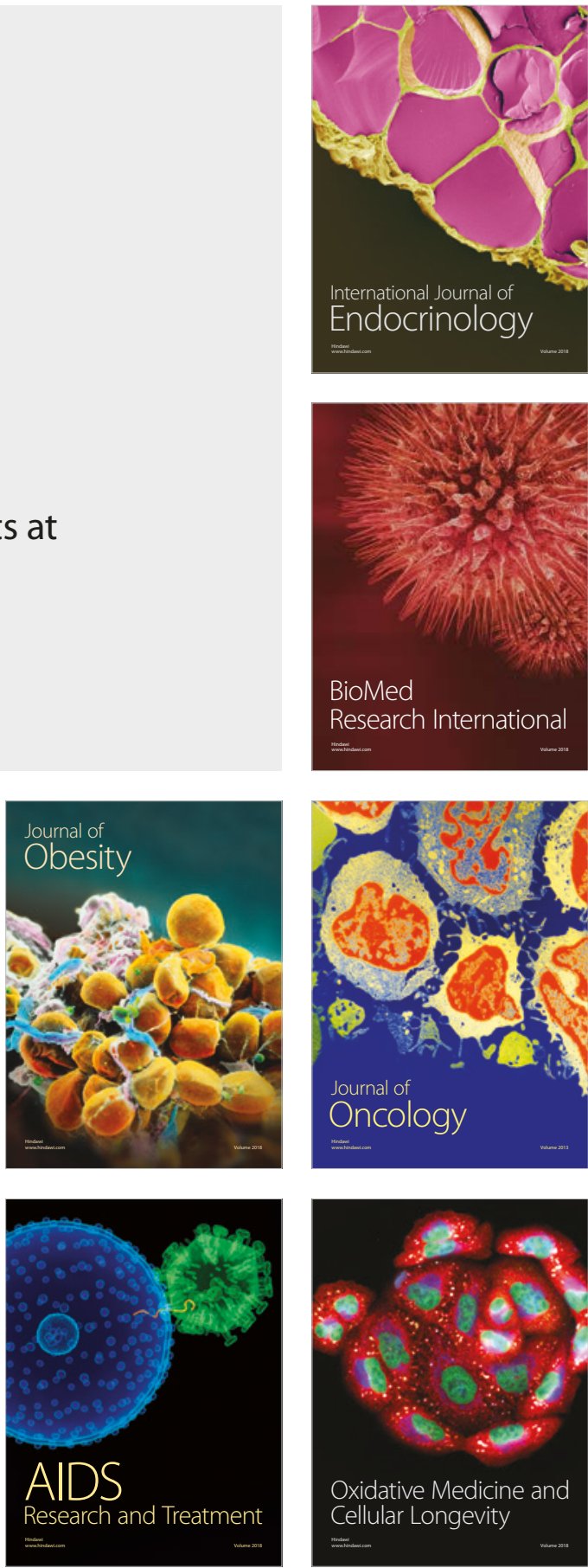